\title{
On the Radioactive Fallout Collected in Tolkyo on 26 th November, 1955
}

\author{
by \\ Y. Sugiura and T. Kanazawa \\ Meteorological Research Institute
}

(Received July 3, 1956)

\begin{abstract}
Artificial radioactivity of rain water and fallout collected in Tokyo around 26th November are described. Next, concerning the radioactive dust fallen on 26 th, the results of radiochemical analysis are presented.
\end{abstract}

\section{Introduction}

It was declared on 23rd November, 1955, by the US Atomic Energy Commission that the largest nuclear weapon test in USSR was carried out on the previous 22nd. Prior to this statement, the artificial radioactivity of $5 \mathrm{~m} \mu \mathrm{C}$ was detected in one liter of rain water collected at this laboratory on 21st, November. Then the activity of as much as $47 \mathrm{~m} \mu \mathrm{C}$ (equivalent to $20,500 \mathrm{cpm}$ ) per $\mathrm{m}^{2}$ per 24 hours was found in fallout on 26th. This was the highest value of artificial radioactivity of fallout which had ever been determined in routine at this laboratory since September, 1954.

The actual intensity of radioactivity of fallout sample available for analysis was about $2000 \mathrm{cpm}^{*}$, which could not be said to be sufficient for analysis. But as it was thought that even with such an intensity of radioactivity as mentioned above, the kinds and contents of radioactive materials existing in fallout might be clarified to some extent, the radiochemical analysis of the dust sample was set up at once.

\section{Radioactivity of rain water and fallout collected around 26 th November}

Before describing the radioactivity of fallout collected on 26th November, the authors call attention to the radioactivity of rain water and fallout which were collected around 26th November. The relevant data are presented in Fig. 1. According to Fig. 1, a higher level of radioactivity was found in rain water on 21st and 27th and in fallout on 29th.

Decay curves for these samples proved that the half life of radioactivity of rain water collected on 27 th and of fallout on 26 th was both about 3 days, while,

* Dust was collected on an area about $1,000 \mathrm{~cm}^{2}$ wide. 


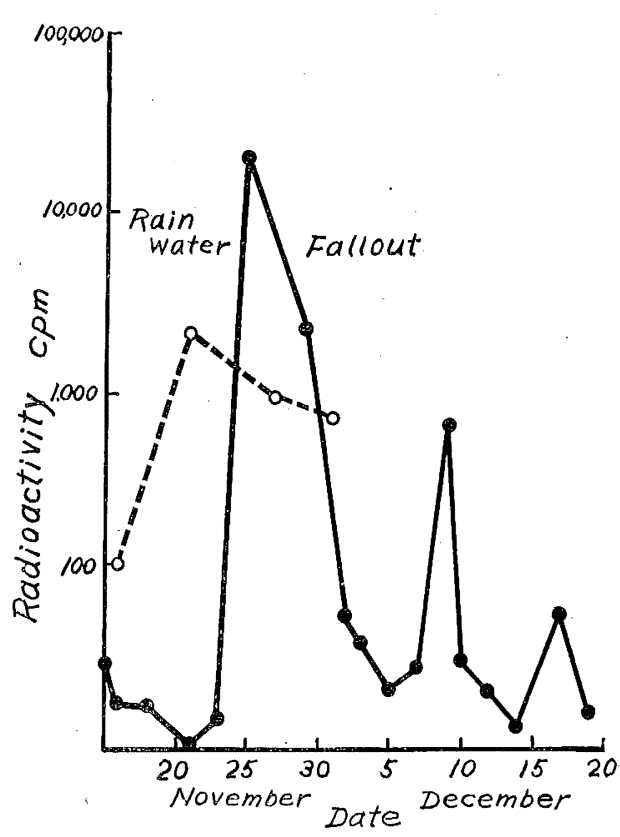

Fig. 1. Radioactivity of rain water and fallout collected in November and December 1955.

\section{Radioactivity of dust fallen on 26 th}

The dust consisted of many sootlike substances and its total amount weighed about $150 \mathrm{mg}$ per $\mathrm{m}^{2}$ per 24 hours. Its radioactivity was $47 \mathrm{~m} \mu \mathrm{C}$ per $\mathrm{m}^{2}$ per 24 hours 4 days after the explosion. The $\beta$ ray energy absorption curve obtained on 28th November is shown in Fig. 2. According to Fig. 2, the energy of the $\beta$ radiation can approximately be classified into three categories and the percentage contribution of each category to the whole of the $\beta$ activity is as follows: on the other hand, the half life of radioactivity of rain water collected on 21st and of the fallout on 29th was both 13 days or so. From this, it may be thought that the radioactivity of fallout collected on 26th and rain water on 27th are due to the explosion of an atomic bomb that took place on 22 nd while the radioactivity of rain water collected on 21st and of the fallout on 29th are due to an explosion before 22nd. It is supposed to be attributed to some meteorological causes that soon after the occurrence of the radioactive rain-out contaminated with fission products derived from the recent explosion, the radioactive rain precipitated which was affected by the explosion carried out previously.

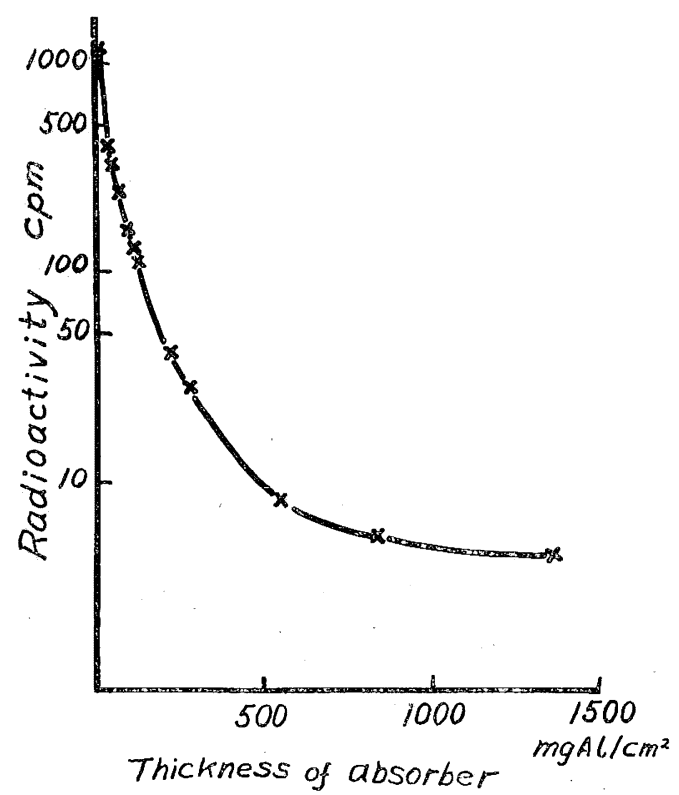

Fig. 2. The $\beta$ ray energy absorption curve for fallout collected on 26th November 1955 .

\begin{tabular}{c|c}
\hline \hline$\beta$-ray energy, Mev & $\begin{array}{r}\text { Percentage of } \beta \text { disintegration rate belonging to } \\
\text { each energy fraction }\end{array}$ \\
\hline 1.2 & 26 \\
0.35 & 34 \\
0.20 & 40 \\
\hline
\end{tabular}




\section{Analytical results of the radioactive dust fallen on 26 th}

An analytical method will briefly be described. Dust was transferred to a porcelain crucible (capacity $25 \mathrm{cc}$ ) and ignited to ashes. The residue was boiled in a diluted hydrochloric acid $(1: 1)$ solution and the radioactive substances were dissolved. The solution was diluted with distilled water to obtain a $0.2 \mathrm{~N}$ hydrochloric acid solution and poured into a cation exchanger (Amberlite IR-120 of ca. $15 \mathrm{cc}$ in volume was used, the size of the resin particles ranging from 100 to 150 meshes) which was preliminarily treated with a diluted hydrochloric acid solution. The succeeding procedure was practiced according to the ordinary method [1]. Fig. 3 shows the elution curves for each group of radioisotopes separated by

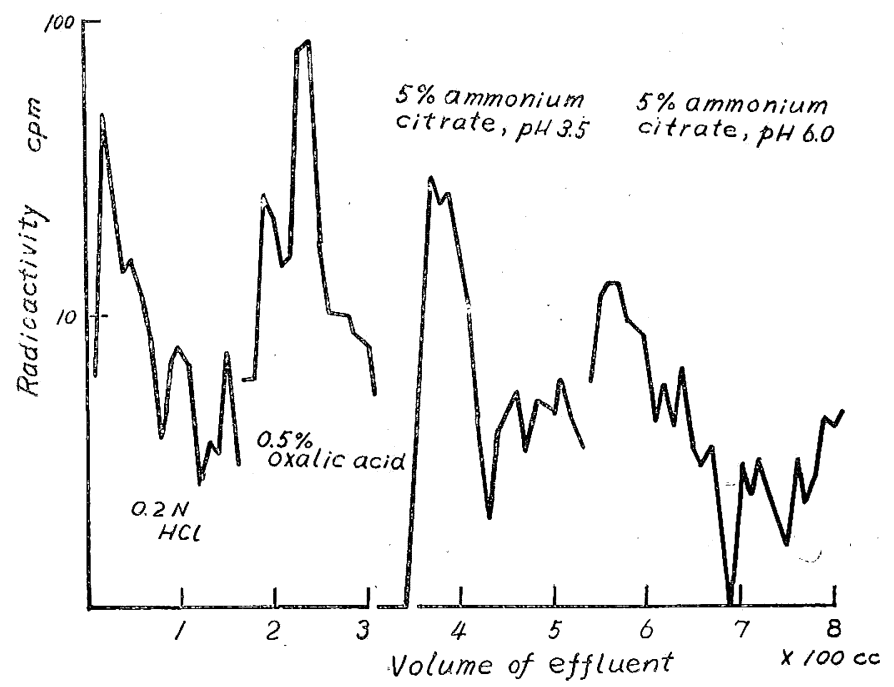

Fig. 3. Elution curves for radioactive dust collected on 26th November 1955.

cation exchanging process. This figure shows that the intensity of radioactivity of the fraction eluted with an oxalic acid solution was the most evident. Since the existence of zirconium, niobium, uranium and neptunium was expected in this fraction, the separation of zirconium and niobium from uranium and neptunium was made in the following method introduced by Y. Yokoyama, Yokohama University [2] :

Fraction of effluent with a $0.5 \%$ oxalic acid solution

\begin{tabular}{|c|c|c|c|}
\hline \multirow[b]{2}{*}{$\begin{array}{l}\text { Fraction passing through } \\
\text { without being absorbed } \\
\text { in resin }\end{array}$} & \multirow[b]{2}{*}{$\begin{array}{l}\text { Fraction of effluent with } \\
2 \mathrm{~N} \mathrm{HNO}_{3}\end{array}$} & $\begin{array}{l}\mathrm{e}, \\
\text { for } 1 \mathrm{hr}\end{array}$ & \multirow[b]{2}{*}{$\begin{array}{l}\text { effluent with } \\
\text { c acid }\end{array}$} \\
\hline & & $\begin{array}{l}\text { Fractior } \\
0.5 \% \text { os }\end{array}$ & \\
\hline
\end{tabular}

The results of the separation made under the above scheme are shown in Fig. 4. Seeing Fig. 4, it is clear that the radioactivity was stronger in the 


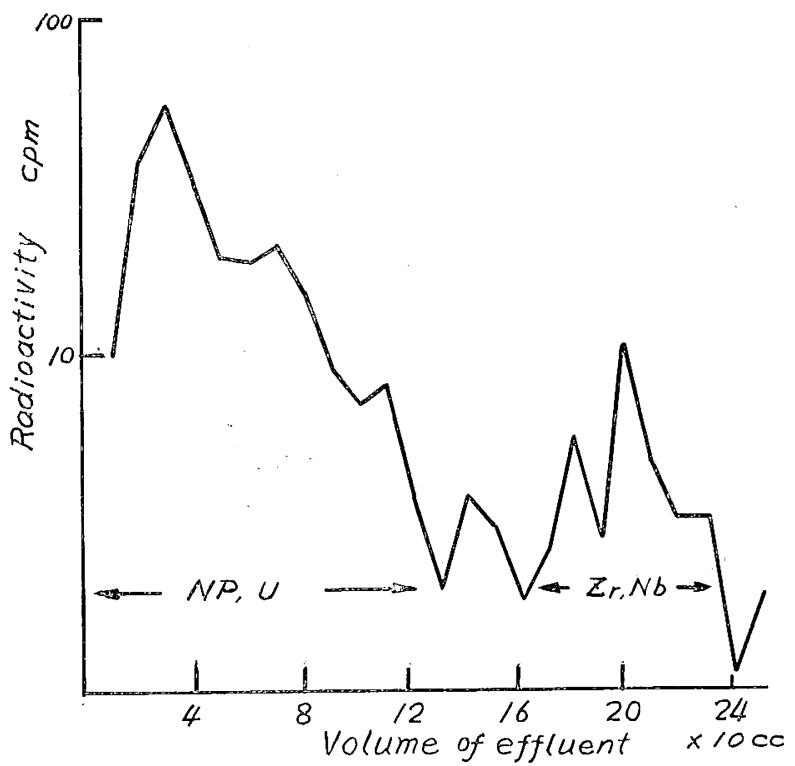

Fig. 4. The Elution curve for the effluent of $\mathrm{NP}, \mathrm{U}, \mathrm{Zr}$ and $\mathrm{Nb}$.

uranium and neptunium fraction than in the zirconium and niobium one. In order to separate uranium and neptunium, the following procedure was followed:

The fraction of effluent with $\mathrm{HNO}_{3}$

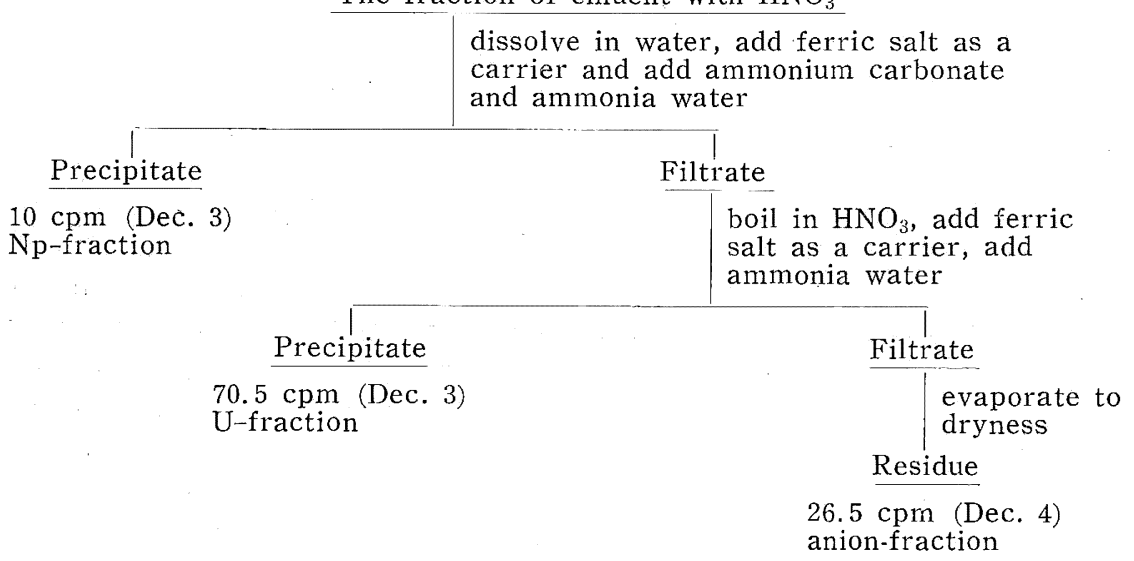

Date in parentheses shows the time when the counting rate of each part was measured.

Since the radioactivity of the neptunium was nearly at the lowest level which could be measured with certainty, it was difficult to identify neptunium by measurement of decay and $\beta$ ray energy absorption but it was thought to be neptunium owing to its chemical speciality. As regards the uranium fraction, it was confirmed as shown in Fig. 5 by both its decay and its $\beta$ ray energy absorption curve 
that the half life was 6.5 days and the maximum energy of $\beta$ ray $0.3 \mathrm{Mev}$. On the other hand, the literature values for U-237 are 6.8 days and $0.23 \mathrm{Mev}$. Accordingly it could be surely concluded as U-237.

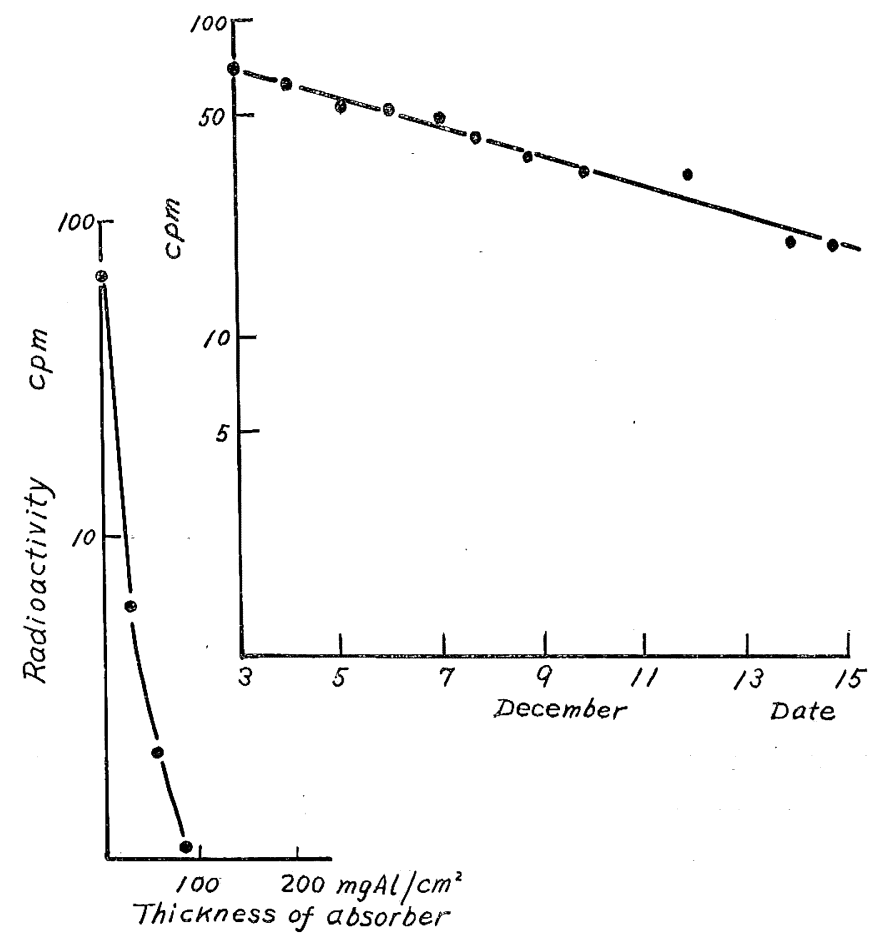

Fig. 5. Decay curve and $\beta$ ray energy absorption curve for uranium fraction.

The radioactivity of $26.5 \mathrm{cpm}$ was detected in the filtrate obtained after the elimination of uranium fraction. As its half life was about 70 hours, it was considered as Te-132. In the fraction of effluent with a $0.2 \mathrm{~N}$ hydrochloric acid solution, as shown in Fig. 6, two sorts of radioisotopes having a half life of 30 hours and 70 hours and some radioisotopes having a longer half life were detected. Since two sorts of radioisotopes having their maximum energies of about 0.2 and 1.5 Mev were detected at the same time, the possible existence of Te-132 and Te-131 (m) was inferred.

Fig. 7 shows the decay curve and the $\beta$ ray energy absorption curve concerning the fraction of effluent eluted with ammonium citrate solution of which the hydrogen ion concentration was preliminarily regulated to $3.5 \mathrm{in} \mathrm{pH}$ scale. Fig. 7 suggests the existence of a radioisotope having a half life of 36 hours and the maximum $\beta$ ray energy of about $1.2 \mathrm{Mev}$ and the existence of others having a half life of ten days or more. As a radioisotope equivalent to the former, La-140 (40 hrs, $\beta^{-} 1.4 \mathrm{Mev}$ ) or Ce-143 (33 hrs, $\beta^{-} 1.35 \mathrm{Mev}$ ) was pointed out.

Fig. 8 shows the decay curve and the $\beta$ ray energy absorption curve concerning to the fraction of effluent with ammonium citrate solution of which $\mathrm{pH}$ was 


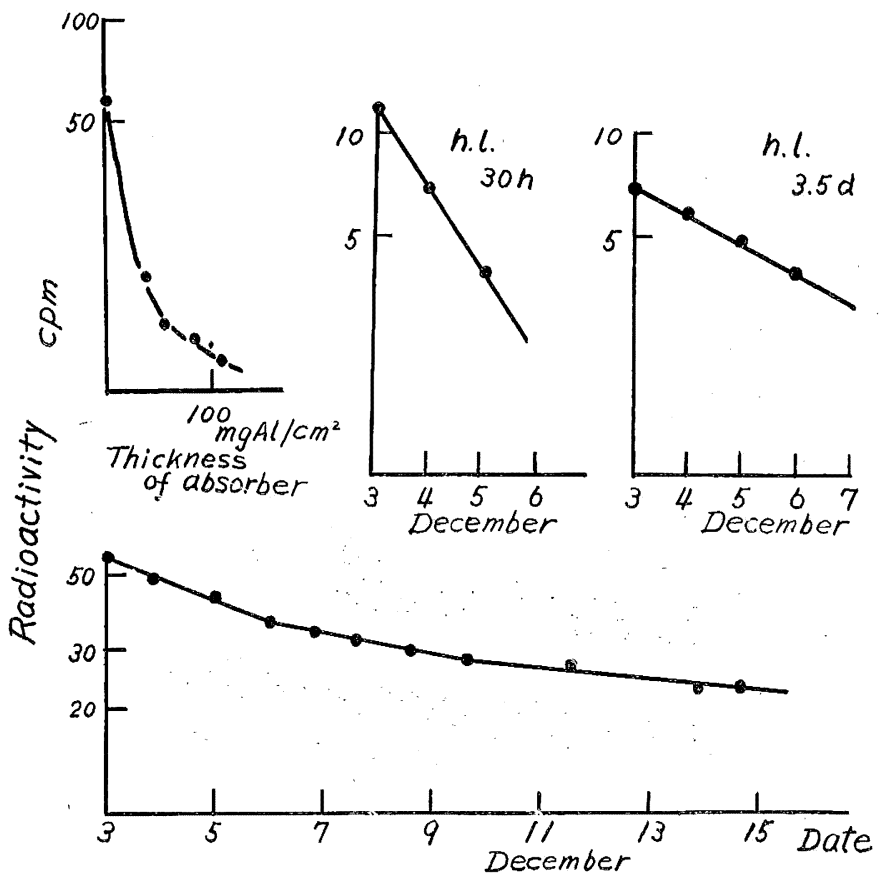

Fig. 6. The $\beta$ ray energy absorption curve and decay curves for $\mathrm{HCl}$ effluent.

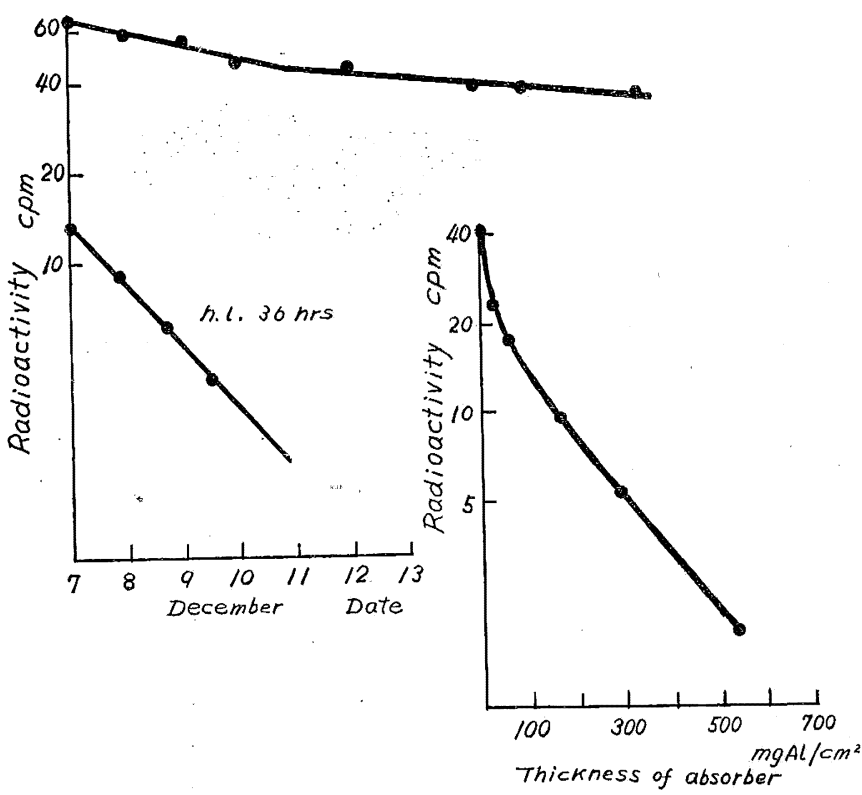

Fig. 7. Decay curve and $\beta$ ray energy absorption curve for the effluent from ammonium citrate solution. 

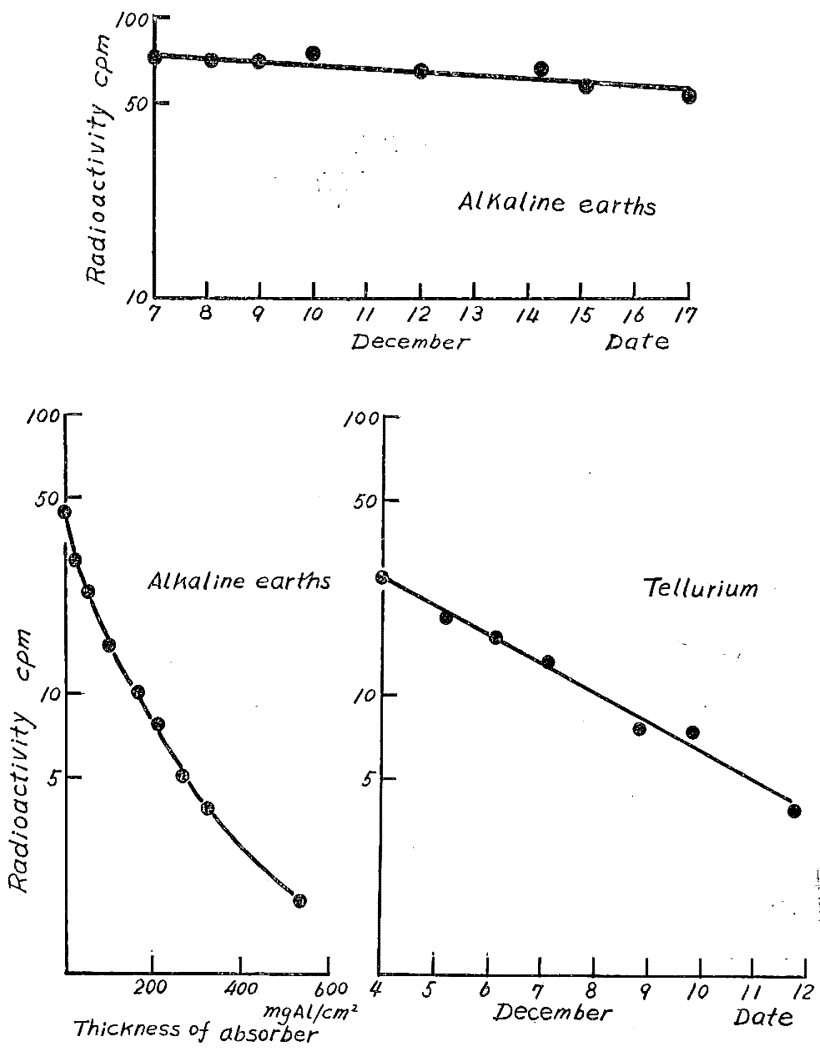

Fig. 8. Decay curves for alkaline earths and tellurium and $\beta$ ray energy absorption curve for alkaline earths.

regulated to 6.0. Fig. 8 suggests the possible existence of a radioisotope having the outward looking half life of about 20 days and the maximum energy of about 1 Mev. Perhaps it may be a mixture of Be-140 and Sr-89. Summarizing the above-mentioned results we obtained Table 1.

Table 1. Analytical results of radioactive dust fallen in Tokyo on 26th November 1955.

\begin{tabular}{l|c}
\hline \multicolumn{1}{c|}{ Radioisotope } & $\begin{array}{r}\text { Percentage of disintegration rate determined } \\
\text { on 3rd December, }\end{array}$ \\
\hline Te-131 (m) & 3.7 \\
Te-132 & 15.0 \\
other anions & 11.5 \\
Zr, Nb & 4.3 \\
La-140, Ce-143 & 13.8 \\
other rare earths & 9.7 \\
alkaline earths & 11.3 \\
U-237 & 27.0 \\
Np & 3.8 \\
\hline
\end{tabular}




\section{References}

[1] K. Kimura et al, 1954: Radiochemical Analysis of "Bikini Ashes" fallen on Board the No. 5 Fukuryu Maru on March 1, 1954. Japan Analyst, 3, p. 335.

[2] Y. Yoкоуама, 1955: Neptunium in radioactive rain water. Proceedings of the Symposium Concerning the Chemistry of Radioactive Rain (the meeting was held at the Meteorological Research Institute in April 1955), 1, p. 1. 